\title{
A Comprehensive Analysis of a Heavy Precipitation Event in Chengdu Plain (China) Based on Ground-Based GPS
}

\author{
Wang Hao ${ }^{1,}$, Wang Yue ${ }^{2}$, Wang Yongqian ${ }^{3}$ \\ ${ }^{1}$ College of Meteorological Observation, Chengdu University of Information Technology, Chengdu, China \\ ${ }^{2}$ Meteorological Service Center, Chengdu Meteorological Bureau, Chengdu, China \\ ${ }^{3}$ College of Resources and Environment, Chengdu University of Information Technology, Chengdu, China
}

Email address:

wh@cuit.edu.cn (Wang Hao)

*Corresponding author

\section{To cite this article:}

Wang Hao, Wang Yue, Wang Yongqian. A Comprehensive Analysis of a Heavy Precipitation Event in Chengdu Plain (China) Based on Ground-Based GPS. Earth Sciences. Vol. 5, No. 4, 2016, pp. 48-55. doi: 10.11648/j.earth.20160504.11

Received: August 3, 2016; Accepted: August 11, 2016; Published: August 31, 2016

\begin{abstract}
This study utilized the ground-based GPS water vapor monitoring network in the Chengdu Plain $\left(102.9^{\circ}-104.9^{\circ} \mathrm{E}\right.$, $\left.30.1^{\circ}-31.4^{\circ} \mathrm{N}\right)$, alongside radiosonde data and National Center for Environmental Prediction and National Center for Atmospheric Research (NCEP/NCAR) reanalysis data, to conduct a comprehensive analysis of a heavy precipitation event in this region in 2008. Correlations were found between the GPS precipitable water vapor (GPS-PWV) variations, the actual precipitation in the region, and the physical mechanism for the GPS-PWV variations. The research results indicate that the variation trends in precipitable water vapor had a significant correlation with actual precipitation. The precipitable water vapor increased and decreased significantly before and after the precipitation event, respectively. The residence time of precipitable water vapor at high levels was correlated with the duration of actual precipitation to some extent. The maximum value of the precipitation intensity lagged behind the precipitable water vapor peak, which brought forward precipitation to a certain degree. A strong ascending motion of the air was linked to increases in PWV, and the intensity of the ascending motion was strongly correlated with GPS-PWV. Different atmospheric thermodynamic conditions also had a notable effect on GPS-PWV variations.
\end{abstract}

Keywords: Ground-Based GPS, Precipitable Water Vapor, Heavy Precipitation, Dynamic Conditions, Thermodynamic Conditions

\section{Introduction}

Water vapor is not only the primary driving force of weather and climate change, but also a key factor in the formation and evolution of disastrous weather. Water vapor plays a vital role in Earth's climate system, and is the key monitoring parameter for local and even global climate change [1]. The content of water vapor in the atmosphere, namely atmospheric precipitable water vapor, represents the amount of actual precipitation converted from the total amount of water vapor contained in a unit area of the atmospheric column, and variations in this parameter often indicate the input and output conditions for regional water vapor, thus playing a vital indicative role in precipitation forecasts, artificial weather modification, assessments of regional water circulation, and analyses of global warming [2]. Furthermore, a basic component of weather forecasting is accurately acquiring the spatial distribution and temporal variation features of water vapor in the atmosphere [3]. Chengdu Plain is located in Sichuan Basin, in the east section of the Qinghai-Tibet Plateau, and belongs to a region with a subtropical humid monsoon climate. The surrounding region is complex in terrain, rich in water vapor, and changeable in weather, with obvious regional climatic features, notably the frequent occurrence of local heavy rains, that may stimulate or intensify disastrous processes such as heavy rains and floods in the middle and lower reaches of the Yangtze River [4]. Therefore, in-depth studies on the variations of atmospheric water vapor and application-oriented experiments on water vapor warming and forecasting are of great scientific significance and societal value for this region.

Water vapor has more complex temporal and spatial variation features than greenhouse gases like carbon dioxide, 
methane, and ozone, and so is more difficult to measure [5], which results in limited applications for water vapor data in meteorology. Since the 1990s, it has become popular practice to employ GPS to estimate the total atmospheric precipitable water vapor, as this is a new potential monitoring technology with great practical value [6]. In recent years, with the rapid development of GPS, its application to the monitoring of water vapor has drawn increased attention. GPS has played an important role in short-term and imminent forecasts, data assimilation, and mode correction as an independent data source, because of its high precision, high temporal-spatial resolution, low cost, and all-weather applicability [7]. In a heavy precipitation forecast and monitoring application, Li et al. [8] utilized the results of heavy rain observations based on GPS, and found that PWV will remain constant for a long period before a heavy rain event, and will increase and decrease before and after the heavy rain event, respectively. Ye et al. [9] conducted analyses and statistical work on GPS-PWV variation features for different severe convective weather systems, concluding that the GPS-PWV threshold value for precipitation in the main rainy season is larger than $50 \mathrm{~mm}$, and the threshold value for severe convective precipitation in this season is greater than $60 \mathrm{~mm}$. Li et al. [3] analyzed the two primary systems (the Plateau Vortex and the Southwest Vortex) that affect precipitation in the southern region of China. They found that before the development of the Plateau Vortex and the Southwest Vortex, GPS-PWV has already presented a rapidly increasing trend, and precipitation predominantly occurs in the period when GPS-PWV is at a high level. Radhakrishna et al. [10] found that the occurrence of convection could be predicted three hours in advance through monitoring GPS-PWV variations. Moore et al. [11] employed an intensive GPS water vapor monitoring network, which plays an important role in timely announcements of flood and debris flow conditions, and is a good supplement to mathematical forecast results within one to six hours of an event. With the development of GPS, the density of the observational network is increasing continuously. To make comprehensive assessments of water vapor information, researchers have gradually moved away from single-station atmospheric precipitable water vapor data toward multi-level and multi-zone water vapor information. With reference to the successful application of tomography to various disciplines and fields [12], appropriate observational matrices can be established through acquiring the water vapor information over multiple slant paths, allowing the 3D spatial distribution of water vapor fields to be theoretically obtained [13]. However, there are some deficiencies in the application of this technology to the inversion of 3D water vapor fields, namely insufficient observational information at lower altitudes and unstable results, which can result in insufficient precision for the requirements of meteorological applications. Hence, the technology is still at the stage of theoretical research.

For current scientific research, the total atmospheric precipitable water vapor inverted using GPS data (GPS-PWV) is extensively applied, while most studies on GPS-PWV focus on the correlation between the evolutional features of the precipitation and precipitable water vapor. The dynamic and thermodynamic conditions involved in weather processes are key points that should not be neglected. To apply GPS water vapor data to practical forecasts effectively, this study utilized the precipitable water vapor data inverted using the ground-based GPS observational network in the Chengdu region, alongside other observational data, to conduct a comprehensive analysis of a continuous heavy precipitation event that occurred on September 22 to 27, 2008. Combined with the dynamic and thermodynamic conditions during the precipitation event, this study aims to investigate the correlation between the evolution of GPS precipitable water vapor and the actual precipitation event, to provide an additional reference for practical forecasts.

\section{Data and Method}

The data used in this study are from Chengdu's regional GPS monitoring network, which was built by the Chengdu Meteorological Bureau and Chengdu Institute of Survey and Investigation in August, 2007. The monitoring network consists of six GPS monitoring stations: Chengdu Station (CDKC), Dujiangyan Station (DUJY), Jintang Station (JITA), Longquan Station (LOQU), Dayi Station (DAYI), and Pujiang Station (PUJI) (Fig. 1). The GPS water vapor monitoring system predominantly consists of receivers and servers. Since relevant meteorological data is needed for the water vapor inversion, all the ground-based GPS monitoring stations have access to the corresponding data from surface weather stations (the data from the six-element automatic weather station developed by the China Huayun Group were adopted for surface meteorological observations). The receivers in the GPS water vapor monitoring system predominantly collect the original GPS observational data and meteorological observational data, and the servers consist of data servers, resolving servers, and application servers. The data servers receive the relevant meteorological data measured by the automatic weather stations, including temperature, pressure, and humidity, receive the signal sent by GPS satellites to generate corresponding files with the "RINEX" extension that can be recognized by the resolving software application, and save and transmit the obtained data. The resolving servers are mainly responsible for resolving retardation and inverting PWV, and the application servers are used for tasks relating to the generation of relevant products for meteorological applications [14].

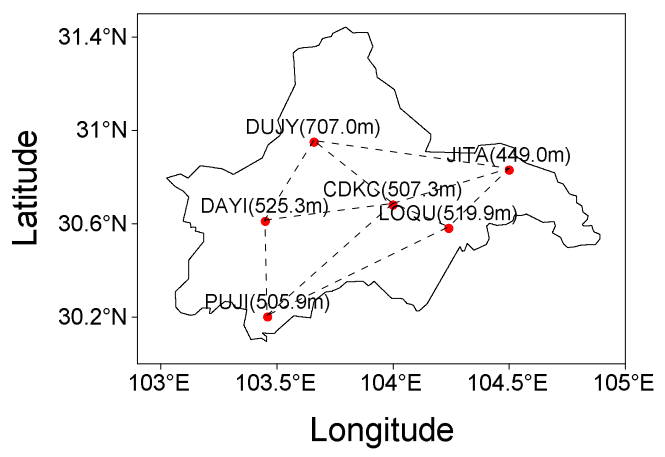

Figure 1. Geographical location distribution and altitudes of the GPS monitoring stations in the Chengdu region. 
The principle of acquiring atmospheric precipitable water vapor from GPS data is to invert the delay in the GPS signal between the atmosphere and the ground receivers. This delay predominantly consists of the ionospheric delay (ID) and the zenith total delay (ZTD). Dual frequency GPS receivers (American Trimble) were adopted in this study for ground-based GPS observations. Since ID is inversely proportional to the square of the signal frequency, the dual frequency receivers can eliminate the linear combination of the atmospheric delay equations of the two frequencies so as to exclude the influence of delay caused by the ionosphere when acquiring the GPS observational data. The ZTD is composed of the zenith hydrostatic delay (ZHD) and the zenith wet delay (ZWD), and is approximately $2.5 \mathrm{~m}$. Over $90 \%$ of the total delay (c. $2.2 \mathrm{~m}$ ) is the hydrostatic delay caused by dry air and approximately $10 \%$ (c. $0.3 \mathrm{~m}$ ) is the wet delay caused by water vapor [15]. The ZHD can be estimated by the ground pressure. Under static equilibrium conditions, the estimation of ZHD can be more precise than $1 \mathrm{~mm}$ if the measurement accuracy of the ground pressure is $0.5 \mathrm{hPa}$ [16]. In this way, wet delays at the mm level can be obtained, and atmospheric PWV can be acquired through formula transformation. The detailed calculation process is as follows:

$$
P W V=\prod \times Z W D .
$$

PWV is obtained by converting the total water vapor content of the atmospheric column to an equivalent height of the liquid water column. The conversion factor $\prod$ is given by the following formula:

$$
\Pi=\frac{10^{6}}{\rho_{w} R_{v}\left(k_{3} / T_{m}+k_{2}^{\prime}\right)}
$$

where $k_{3}=3.739 \times 105 \mathrm{~K} 2 \cdot \mathrm{hPa}-1$ and $k_{2}^{\prime}=22 \mathrm{~K} \cdot \mathrm{hPa}-1$ are experimental constants; $\mathrm{Rv}=461.495 \mathrm{~J} \cdot \mathrm{kg}-1 \mathrm{~K}-1$ is the specific gas constant of water vapor; Tm is the atmospheric weighted average temperature, representing the integral of the water vapor pressure and absolute temperature over the observation station, and is expressed as

$$
T_{m}=\frac{\int_{z}\left(p_{v} / T\right) d z}{\int_{z}\left(p_{v} / T^{2}\right) d z}
$$

where $P_{v}$ and $T$ represent water vapor pressure and temperature, respectively. $T_{m}$ is usually obtained by employing the approximate calculation formula (4) proposed by Bevis et al. [17], or by establishing a local $T_{m}$ formula based on years of sounding data [18].

$$
T_{m}=70.2+0.72 T_{s}
$$

To verify the rationality and accuracy of the GPS-PWV data, the GPS-PWV data from CDKC for autumn 2008 were selected, and the atmospheric precipitable water vapor data inverted for the sounding station (longitude: $103.83^{\circ}$; latitude: $30.70^{\circ}$; altitude: $540.6 \mathrm{~m}$ ) near the GPS observation station over the same interval (Radiosonde-PWV) were also selected. The obtained PWV can be calculated using (5), as shown below.

$$
P W V=-\frac{1}{g} \int_{p=p_{0}}^{p=p_{1}} q d p
$$

where q stands for the specific humidity of the corresponding height (here, the heights are the ground level, and 925, 850, $700,500,400,300$, and $200 \mathrm{hPa}$ ); $p_{0}$ stands for the ground pressure; and $p_{1}$ stands for the atmospheric zenith pressure. The two accuracy indexes, namely the mean absolute error (MAE) and root mean square error (RMSE) were calculated as $3.77 \mathrm{~mm}$ and $4.81 \mathrm{~mm}$, respectively. This level of accuracy meets the requirement for meteorological research and operational applications [19].

This study also employed the radio-sounding data, acquired twice daily (08:00 and 20:00, local time, the same below), and the six-hourly $1^{\circ} \times 1^{\circ}$ reanalysis data provided by NECP/NCAR, featuring long temporal coverage, high spatial resolution, and strong data continuity. The reanalysis data are the comprehensive results from multiple observational data and operational methods, and strict quality control was implemented during the output process, achieving high data reliability [20, 21]. Additionally, the interpolation scheme for missing GPS-PWV data in the Chengdu region proposed by Wang et al. [22] was adopted in this study.

The structure of subsequent presentation of this study's research is as follows: section three provides the results and discussion of the study and mainly presents the general analyses of cases and the study of the corresponding GPW-PWV variation features, as well as their internal physical mechanisms; section four provides the conclusions and future research directions from the study.

\section{Results and Discussion}

\subsection{Case Overview}

The 500hPa circulation situation map for East Asia at 20:00 on September 22, 2008 (Fig. 2) shows that the atmospheric circulation is mainly zonal circulation; the strong subtropical high has extended westward to Sichuan Basin and, compared with the corresponding historical location, its location is further westward and its ridge is further northward. At that time, Chengdu region was located at the edge of the subtropical high. In addition, the wind arrows and potential temperatures in Fig. 2 show that CDKC presents an overall state of "dry in the upper level and wet in the lower level of the atmosphere." The southerly water vapor transportation has reached a higher altitude in the troposphere, and the convective instability in the lower atmosphere caused by surface heating and water vapor has extended to $500 \mathrm{hPa}$, i.e., it has reached an overall convective state below the $500 \mathrm{hPa}$ level. Additionally, the vertical wind direction of the entire atmosphere presents a clockwise rotation from lower to higher levels, which further indicates the presence of warm advection. Under this circulation background and atmospheric 
distribution, the occurrence of local severe convective weather is typical, generating precipitation in the warm region [23].

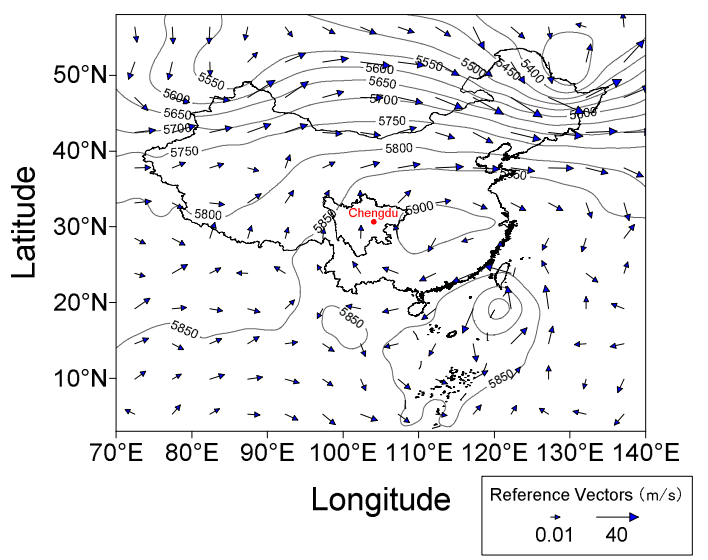

(a)

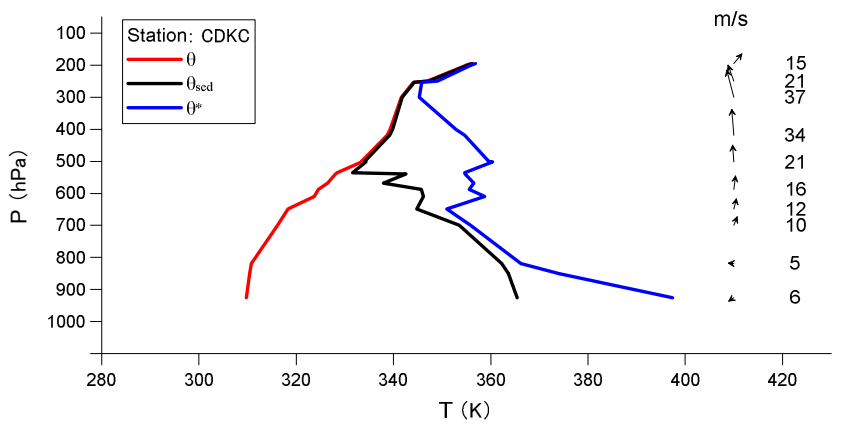

(b)

Figure 2. (a) $500 \mathrm{hPa}$ atmospheric circulation situation and (b) the distribution of wind arrows and potential temperatures at 20:00 on September 22, 2008. The data for (a) are from the National Center for Environmental Prediction (NCEP) $1^{\circ} \times 1^{\circ}$ reanalysis data. The three curves in (b) represent, the potential temperature $\theta$ (red line), the potential pseudo-equivalent temperature $\theta_{\text {sed }}$ (black line) calculated from the dew point temperature, and the potential pseudo-equivalent temperature $\theta^{*}$ (blue line) under saturated conditions.

During the precipitation process, the $500 \mathrm{hPa}$ subtropical high was stable with little motion (Fig. 3a). The typhoon Hagupit landed in Guangdong Province, China (109.6 $117.4^{\circ} \mathrm{E}, 20.1^{\circ}-25.5^{\circ} \mathrm{N}$ ) at 20:00 on September 23, 2008, and moved gradually westward due to the easterly airflow from the south of the subtropical high. Meanwhile, the westward movement of Hagupit increased the stability of the subtropical high. A low-pressure system was generated in the east Qinghai-Tibet Plateau, and moved gradually eastward, influencing the weather of Sichuan Basin. Since the subtropical high was relatively strong, it blocked and slowed the plateau low-pressure system, which was conducive to the continuity of precipitation in the west basin. The southerly wind in the basin at $700 \mathrm{hPa}$ intensified at 20:00 on September 23 to form a low-level jet with a maximum wind speed of about $20 \mathrm{~m} / \mathrm{s}$, which was conducive to water vapor transportation and strengthened the lifting movement. At 20:00 on September 25, it became a shear line in the basin, with the typhoon gradually weakening to a low pressure and moving west to the east of Yunnan Province. The southerly airflow outside the typhoon encouraged the transportation of water vapor from the South China Sea to the basin. An easterly airflow remained at $850 \mathrm{hPa}$, which was conducive to the backflow of cold air that influenced the weather of the basin, and the precipitation intensity was increased owing to orographic lifting. According to the distribution of wind arrows and potential temperatures in the Fig. $3 \mathrm{~b}$, the unstable energy over CDKC remained high, and the humidity at the middle and high levels increased, encouraging the occurrence and continuity of heavy rains.

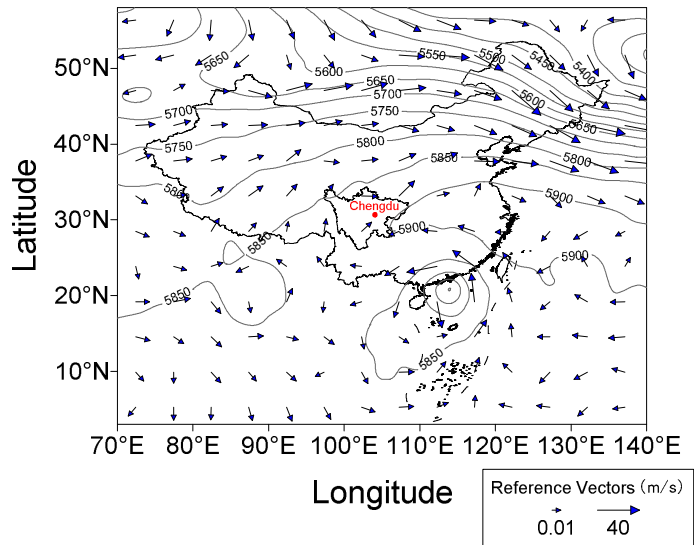

(a)

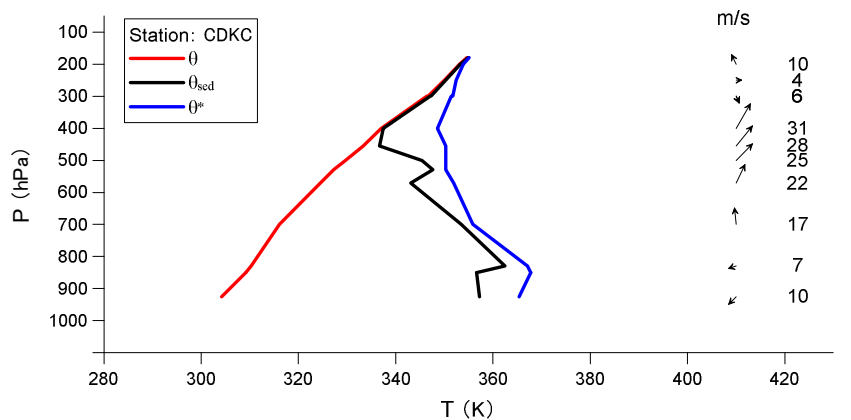

(b)

Figure 3. (a) $500 \mathrm{hPa}$ atmospheric circulation situation for East Asia and (b) the distribution of wind arrows and potential temperatures at 20:00 on September 23, 2008

\subsection{Analysis of the Evolution of GPS-PWV and Precipitation}

From September 22 to 27 in 2008, the variation trends in the atmospheric precipitable water vapor and the timing of precipitation reflected by the GPS-PWV data from each station remained basically consistent (although some differences were observed at DUJY), indicating that the atmospheric water vapor was evenly distributed over most of the regions in Chengdu Plain. The average daily precipitable water vapor from the five observation stations was $48.25 \mathrm{~mm}$, with the maximum and minimum values at PUJI $(51.87 \mathrm{~mm})$ and LOQU (46.46 mm), respectively. According to the actual precipitation distribution, all the observation stations present three obvious precipitation events, but their occurrence times 
and intensities differ. Since the variation trends of the precipitable water vapor presented at each observation station are related to the occurrence time of actual precipitation, this study selected CDCK to investigate the relationship between GPS-PWV and actual precipitation.

From September 22, GPS-PWV at CDKC remained above $40 \mathrm{~mm}$ (Fig. 4a), far surpassing the average level for the autumn of $2008(27 \mathrm{~mm})$ [2], indicating that there was a large increase in the atmospheric water vapor content at the station. From 03:00 of September 23, GPS-PWV gradually increased from around $40 \mathrm{~mm}$ to above $50 \mathrm{~mm}$, and the first precipitation event occurred in Chengdu; this was a showery precipitation event, as it did not last for a long period, although its intensity was large at some points (the 30 -min precipitation value exceeded $20 \mathrm{~mm}$ ). At the end of the first precipitation event, GPS-PWV also dropped rapidly.

From 18:00 on September 23, GPS-PWV increased rapidly. Its increasing amplitude and intensity were far greater than the first PWV increase (Fig. 4b). GPS-PWV at CDKC soared from 18.24 $\mathrm{mm}$ to $61.55 \mathrm{~mm}$ at a rate of $2.4 \mathrm{~mm} / \mathrm{hr}$. However, it should be noted that during the rapid increase of GPS-PWV, the rate of the increase slowed when GPS-PWV exceeded $50 \mathrm{~mm}$, and when GPS-PWV exceeded $50 \mathrm{~mm}$, a small amount of precipitation occurred. GPS-PWV continued to present narrow shocks at the high level and the precipitation also increased gradually. At approximately 08:30 on September 24, the 30-min precipitation level reached $42.21 \mathrm{~mm}$. When the maximum precipitation occurred, GPS-PWV dropped rapidly. The periodical rapid increases in GPS-PWV were found to be closely associated with precipitation, especially peak precipitation, and the rapid increases in GPS-PWV preceded the actual precipitation.

After the end of the primary precipitation event in the second stage, GPS-PWV also dropped to some extent, but the intensity and amplitude of the drop was smaller than that seen in the first stage. Over time, PWV gradually rose again, but at a slower rate and with a smaller amplitude. The corresponding actual precipitation during this period also reduced significantly. For example, the 30-min precipitation at CDKC reduced to less than $10 \mathrm{~mm}$. The difference between this precipitation event and the previous two events is that GPS-PWV remained at a high level for a long period while sustaining small-amplitude spikes, and the corresponding duration of the precipitation was also longer than that of the previous two events. The precipitation event tended to end with another drop in GPS-PWV.

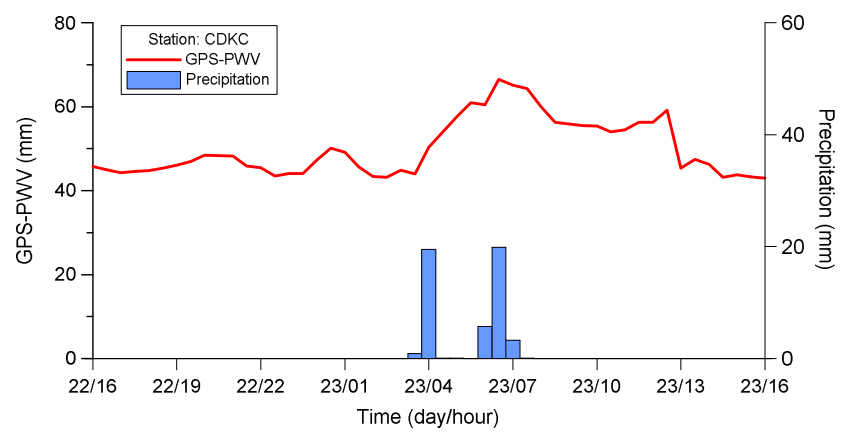

(a)

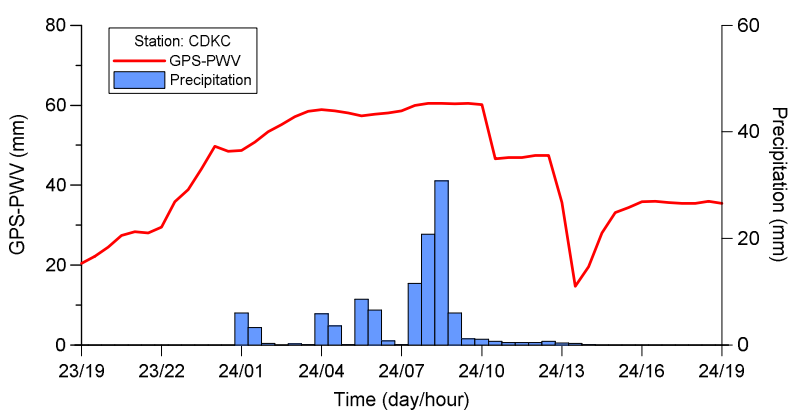

(b)

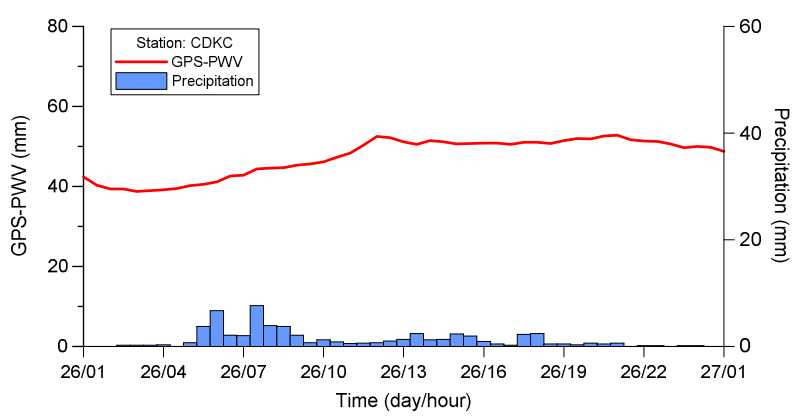

(c)

Figure 4. Variation trends of the ground-based GPS-PWV and the precipitation at $C D K C$ with time. (a) First-stage precipitation event, (b) second-stage precipitation event, and (c) third-stage precipitation event.

\subsection{Analysis of the Correlation Between GPS-PWV and Dynamic and Thermodynamic Conditions}

From a qualitative perspective, the above analyses show that GPS-PWV has a strong correlation with actual precipitation, but it is difficult to determine a linear relationship between the variation of GPS-PWV and actual precipitation. Since the change in GPS-PWV usually precedes actual precipitation, the internal physical mechanism of the GPS-PWV variation requires further analysis and research for forecasts and scientific research.

Generally speaking, saturated water vapor is only one of the essential conditions for the occurrence of precipitation. Precipitation will not occur easily if the corresponding dynamic and thermodynamic conditions are not met [7]. To determine the internal cause of the GPS-PWV variation, which has great significance for practical weather forecasts, this study conducted further analysis and research of the relevant dynamic and thermodynamic conditions of the process, in order to understand their relationship with GPS-PWV.

Since the variation trends of GPS-PWV and actual precipitation for each observation station in this precipitation process were basically identical, CDKC was selected as representative for an analysis of the dynamic and thermodynamic conditions in the precipitation process, to further explain the occurrence and developmental mechanisms of the precipitation process. It was found, through analysis of the vertical velocity field in the three precipitation events at CDKC (Fig. 5), that from 16:00 on September 22 (Fig. 5a) an ascending motion occurred in the lower levels over the station, which provided the dynamic environment for the accumulation of GPS-PWV. At 03:00 on September 23, the ascending motion extended upward from the 
lower and middle levels to $200 \mathrm{hPa}$. Meanwhile, two ascending centers were formed at lower levels and higher levels, respectively. By comparing the states of GPS-PWV at that time and this time point, it can be found that conditions were consistent with those for the first stage when GPS-PWV increased rapidly, suggesting that a strong vertical ascending motion was conducive to the gathering of water vapor, and may represent the physical mechanism behind the rapid variation of GPS-PWV. The ascending motion in the second stage (Fig. 5b) had a more obvious correlation with GPS-PWV. From 12:00 on September 23, the strong ascending motion extended to higher levels constantly, with the maximum central intensity reaching $1.4 \mathrm{~Pa} / \mathrm{s}$. At that time, the heaviest precipitation corresponded with the strongest ascending motion. After 10:00 on September 26 (Fig. 5b), the ascending motion became a descending motion over the observation station, which hindered the transportation of water vapor, and correspondingly the precipitation in the third stage also weakened gradually, with a dramatic decrease in the precipitation intensity and amplitude, further indicating that the intensity of precipitation was directly affected by the maximum speed of the vertically ascending air and the central intensity of the vertical speed.

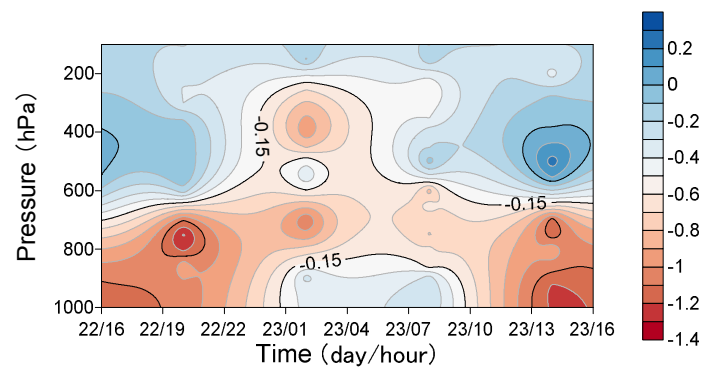

(a)

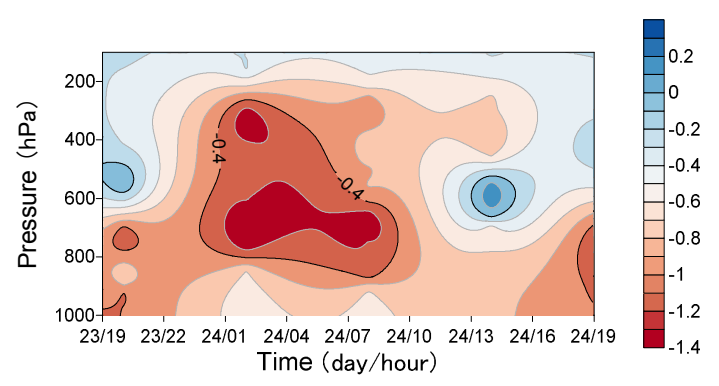

(b)

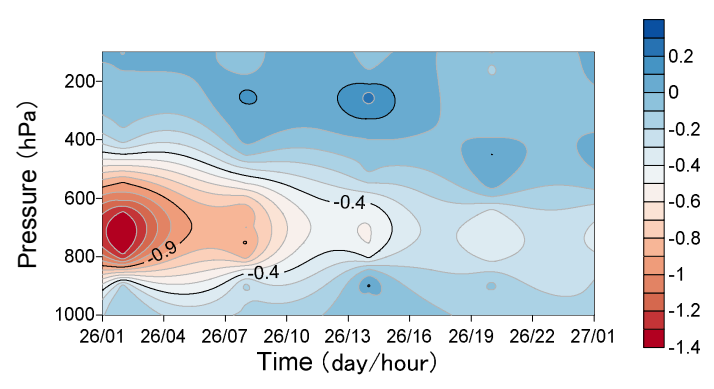

(c)

Figure 5. Vertical cross section of the distribution of vertical wind speed over CDKC with time (unit: $P a \cdot \mathrm{s}^{-1}$ ). (a) First-stage precipitation event, (b) second-stage precipitation event, and (c) third-stage precipitation event.
The atmospheric thermodynamic conditions are also a key factor that influences the precipitation process. This study investigated the atmospheric thermodynamic variation features before and after the heavy precipitation event through the vertical distribution of potential pseudo-equivalent temperature $\theta$ se at $\mathrm{CDKC}$ during this precipitation event. The vertical distribution of $\theta$ se can reflect the convective stability of the atmosphere: when the potential pseudo-equivalent temperature decreases with height $\left(\frac{\partial \boldsymbol{\theta}_{s e}}{\partial z}<0\right)$, this indicates that air is ascending throughout the atmosphere, with the atmosphere presenting the feature of potential instability; in contrast, if $\theta$ se increases with height $\left(\frac{\partial \boldsymbol{\theta}_{s e}}{\partial z}>0\right)$, this suggests the stability of the total atmospheric energy, with the atmosphere presenting a stable atmospheric state [7].

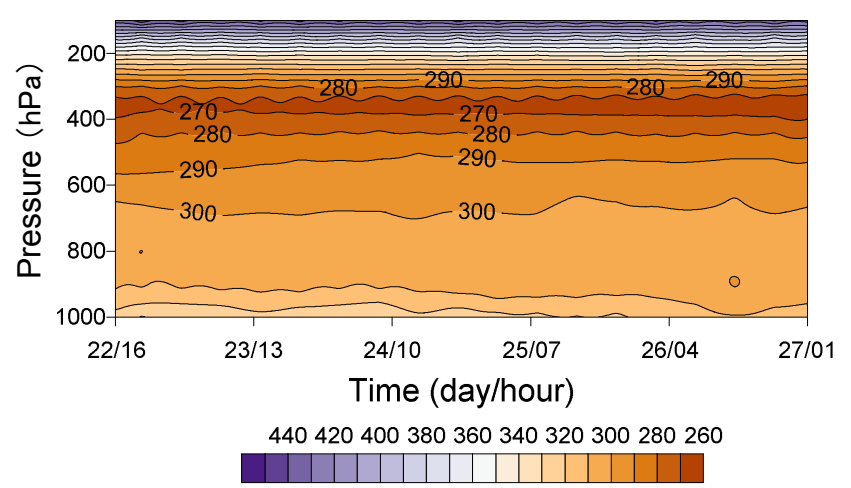

Figure 6. Cross section of the distribution of the potential pseudo-equivalent temperature $\theta_{\text {se }}$ (unit: $K$ ) with time at $C D K C$.

The atmosphere above CDKC presented a state of instability throughout the entire precipitation stage, and this instability was most obvious at lower levels, apparently representing a convective unstable area (equivalent $\theta$ se intensity, $\frac{\partial \boldsymbol{\theta}_{s e}}{\partial z}<0$ ) (Fig. 6). The duration unstable stage was correlated with the variations in GPS-PWV and actual precipitation to some extent. From 10:00 of September 26, the gradient of the potential pseudo-equivalent temperature at lower levels began to decrease, indicating that the unstable atmospheric state began to weaken, and this weakening corresponded with the weakening of the heavy precipitation event. Additionally, the weakening of the unstable energy further restrained the development of GPS-PWV.

\section{Conclusions and Discussion}

This study conducted a comprehensive analysis of the precipitation process by utilizing ground-based GPS. The research conclusions are as follows.

(1) The variation trends of precipitable water vapor showed a strong correlation with the variation trends of actual precipitation. Before and after the precipitation event, the precipitable water vapor clearly increased and decreased, respectively. The duration over which 
precipitation water vapor remained at high levels was correlated with the duration of actual precipitation to some extent. The longer precipitable water vapor remained at high levels, the greater the accumulation of water vapor and the longer the duration of the corresponding precipitation.

(2) The rapid increase in precipitable water vapor usually occurred before precipitation. The precipitable water vapor reached its maximum when precipitation occurred, and subsequently dropped rapidly. When the precipitable water vapor reduced to a certain value, this suggested that the precipitation event was coming to an end. The maximum value of the precipitation intensity lagged behind the peak of precipitable water vapor, indicating that precipitable water vapor brought forward precipitation to some extent.

(3) Associating GPS-PWV variations with the corresponding dynamic and thermodynamic conditions shows that a strong ascending motion was conducive to increases in PWV, and the intensity of the ascending motion was strongly correlated with the value of GPS-PWV. Different atmospheric thermodynamic conditions also had an effect on GPS-PWV variations.

In summary, the water vapor variation conditions above the stations can be reflected almost in real-time by utilizing the GPS-PWV data at high temporal-spatial resolutions. By combining the dynamic and thermodynamic conditions during the process, the practical application value of GPS water vapor products can be enhanced, and the capacity of the data for monitoring and forecasting the evolution of water vapor can be further improved. However, the above analyses also show that there is still scope for further development in the application of ground-based GPS to water vapor monitoring. For example a critical point for the inversion technology is how to accurately acquire the distribution features of the atmospheric near real-time $3 \mathrm{D}$ water vapor to realize $3 \mathrm{D}$ monitoring of water vapor. Regarding the monitoring method, the addition of other sounding data (such as the data from dual polarized radar) can realize the real-time presentation of water vapor information, dynamic information, and thermodynamic information to facilitate early warning and monitoring of precipitation.

\section{Acknowledgments}

This work was supported by the National Natural Science Foundation of China (No. 41405036, No. 41471305, and No. 41375043), the 2015 Middle-Aged Academic Leaders Research Fund of Chengdu University of Information Technology (CUIT) (J201502), a Project of the Sichuan Education Office (15ZB0181), and the Scientific Research Foundation of CUIT (KYTZ201413, CRF201401). The authors would like to express their sincere thanks to the Chengdu Meteorological Bureau for supplying the GPS-PWV data. The authors also thank the reviewers for their constructive comments and editorial suggestions that significantly improved the quality of the paper.

\section{References}

[1] J. T. Kiehl and K. E. Trenberth, "Earth's annual global mean energy budget,” Bull. Am. Meteor. Soc., vol. 78, pp. 197-208, 1997.

[2] H. Wang, M. We, G. Li, S. Zhou, and Q. Zeng, "Analysis of precipitable water vapor from GPS measurements in Chengdu region: Distribution and evolution characteristics in autumn," Advances in Space Research, vol. 52, pp. 656-667, 2013.

[3] G. Li and J. Deng, "Atmospheric water monitoring by using ground-based GPS during heavy rains produced by TPV and SWV," Adv. Meteorol. 2013, doi:10.1155/2013/793957.

[4] G. Li, D. Huang, J. Guo, G. Li, L. Hao, and H. Wang, Ground-based GPS meteorology, Science Press, Beijing, 2010, pp. 1-346. (in Chinese)

[5] V. Sibylle, D. Reinhard, R. Axel, and F. Mathias, "Validation of precipitable water vapor within the NCEP/DOE reanalysis using global GPS observations from one decade," J. Climate, vol. 23 , pp. $1675-1695,2010$.

[6] M. Bevis, S. Businger, and T. Herring, "GPS Meteorology: Remote sensing of atmospheric water vapor using the Global Positioning System," J. Geophys. Res., vol. 97, pp. 1578715801, 1992.

[7] H. Wang, J. He, M. Wei, Z. Zhang, and S. Tang, "Synthesis analysis of one severe convection precipitation event in Jiangsu using ground-based GPS technology," Atmos., vol. 6 (7), pp. 908-927, 2015.

[8] Y. Li, B. Xu, X. Hu, and P. He, "Test and study of the earth-based GPS technique for remote sensing of atmospheric precipitable water,” J. Appl. Meteor. Sci., vol. 12 (1), pp. 6169, 2001. (in Chinese)

[9] Q. Ye, L. Yang, J. Ding, Y. Lu, X. Xu, H. Yi, J. Zhu, and X. Liu, "Application of GPS/PWV data to forecast strong convection weather in Shanghai," Torrential Rain and Disasters, vol. 27 (2), pp. 141-148, 2008.

[10] B. Radhakrishna, F. Fabry, J. Braun, and T. Hove, "Precipitable water from GPS over the continental United States: diurnal cycle, intercomparisons with NARR, and link with convective initiation,” J. Clim., vol. 28, pp. 2584-2599, 2015.

[11] A. Moore, I. Small, S. Gutman, Y. Bock, J. Dumas, P. Fang, J. Haase, M. Jackson, and J. Laber, "National weather service forecasters use GPS precipitable water vapor for enhanced situational awareness during the southern California summer monsoon,” Bull. Amer. Meteor. Soc., vol. 96, pp. 1879-1894, 2015.

[12] R. Kevin and F. Anthony, "Three-dimensional UAV-based atmospheric tomography," J. Atmos. Ocean. Tech., vol. 30, pp. 336-344, 2013.

[13] L. P. Gradinarsky and P. Jarlemark, "Ground-based GPS tomography of water vapor: analysis of simulated and real data," J. Meteor. Soc. JPN., vol. 82 (1B), pp. 551-560, 2004.

[14] H. Wang and G. Li, "Construction and Application about the Monitoring System of Water Vapor Derived from Ground-based GPS in Chengdu," J. Geo-Info. Sci., vol. 13 (2), pp. 213-218, 2011. (in Chinese) 
[15] C. R. Rocken, H. T. Van, and M. Johnson, "GPS/STORM-GPS sensing of atmospheric water vapor for meteorology," J. Atmos. Ocean. Tech., vol. 12, pp. 468-478, 1995.

[16] G. Elgered, J. L. Davis, and T. A. Herring, "Geodesy by radio interferometry: water vapor radiometry for estimation of the wet delay," J. Geophys. Res., vol. 96, pp. 6541-6555, 1991.

[17] M. Bevis, S. Businger, and T. A. Herring, "GPS Meteorology: Remote sensing of atmospheric water vapor using the Global Positioning System,” J. Geophys. Res., vol. 97, pp. 1578-15801, 1992.

[18] J. Guo, G. Li, and D. Huang, "Establish local model for weighted mean temperature of the troposphere based on 40a radiosonde data in Chengdu and Chongqing region," J. Wuhan Univ. (Inf. Sci.), vol. 33, pp. 43-46, 2008.

[19] H. Wang, M. Wei, G. Li, S. Zhou, and Q. Zeng, "Analysis of precipitable water vapor from GPS measurements in Chengdu region: Distribution and evolution characteristics in autumn," Adv. Space Res., vol. 52, pp. 656-667, 2013.
[20] E. Kalnay, M. Kanamitsu, R. Kistler, W. Collins, D. Deaven, L Gandin, M. Iredell, S. Saha, G White, and J. Woollen, "The NCEP/NCAR 40-year reanalysis project," Bull. Am. Meteorol. Soc. Vol. 77, pp. 437-471, 1996.

[21] H. Wang, M. Wei, and S. Zhou, "A feasibility study for the construction of an atmospheric precipitable water vapor model based on the neural network technology," Desalin. Water. Treat., 52 (37-39), pp. 7412-7421, 2014.

[22] H. Wang, D. Wang, and G. Li, "A method of inserting and mending for the GPS precipitable water vapor," 2011 International Conference on Multimedia Technology, pp. 3350-3353, 2011.

[23] H. Wang, G. Chen, H. Lei, Y. Wang, and S. Tang, "Improving the Predictability of Severe Convective Weather Processes by Using Wind Vectors and Potential Temperature Changes: A Case Study of a Severe Thunderstorm," Adv. Meteorol., DOI 10.1155/2016/8320189, 2016 